\title{
"Nous devons mettre en avant les avantages du DEP»
}

\section{Interview: Bruno Kesseli}

Dr méd. et lic. phil., rédacteur en chef

La loi fédérale sur le dossier électronique du patient (LDEP) est entrée en vigueur le 15 avril dernier. Depuis, plus grand monde ne parle de ce projet d'envergure, qui doit permettre d'améliorer la prise en charge des patients. A l'occasion d'un entretien, Pascal Strupler fait le point sur la mise en œuvre. Le directeur de l'Office fédéral de la santé publique (OFSP) se montre convaincu des avantages du DEP et prend position sur les possibles obstacles.

Monsieur Strupler, si vous assimilez le projet du dossier électronique du patient (DEP) à un marathon, à quelle étape de la course en sommes-nous aujourd'hui?

Pour moi, le marathon a commencé en mai 2013, lorsque le message du Conseil fédéral relatif à la loi fédérale sur le dossier électronique du patient a été transmis au Parlement. Tout ce qui s'est passé avant correspondait plutôt à la phase d'entraînement. L'or-

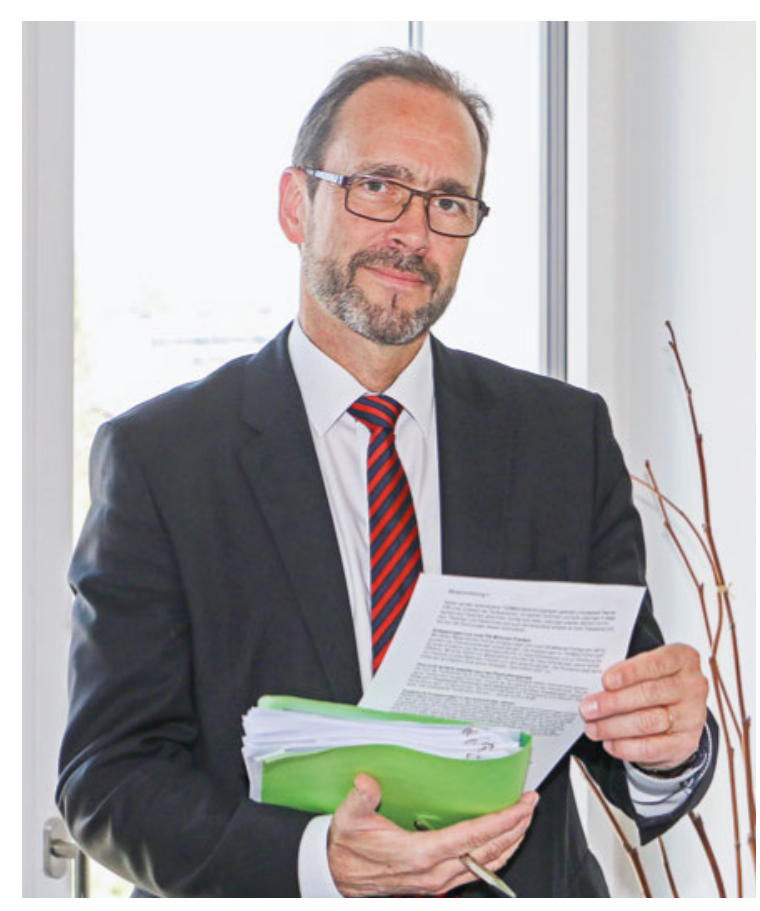

«Nous avons parcouru les trois-quarts du marathon, mais la dernière ligne droite ne s'annonce pas moins difficile»: Pascal Strupler, directeur de I'OFSP au sujet du DEP. gane de coordination entre la Confédération et les cantons, eHealth Suisse, existe déjà depuis 2007. Nous avions déjà effectué un travail de fond et envisagé plusieurs modèles. La loi et ses ordonnances sont en vigueur depuis avril 2017, mais le dossier électronique n'existe bien évidemment pas encore. Je dirais que nous avons parcouru les trois-quarts du marathon, mais la dernière ligne droite ne s'annonce pas moins difficile.

\section{Et en quoi consiste cette dernière ligne droite?}

Il va falloir convaincre et transmettre un grand nombre d'informations à plusieurs niveaux. Et nous devons encore régler beaucoup de détails sur le plan technique et organisationnel. Sans perdre de vue notre objectif.

Cela fait des années que vous et différents experts réfléchissez au DEP. Si l'on se tourne vers les médecins et les patients, on a l'impression que ce sujet est loin d'être une réalité pour eux.

Oui, c'est vrai, je partage votre avis. C'est pourquoi nous avons entrepris, avec eHealth Suisse et les cantons, de mettre au point une sorte de feuille de route que nous allons suivre jusqu'à la création du DEP. Une partie importante du travail consistera à informer. Les médecins et les associations médicales seront notre priorité.

\section{Un groupe assez hétérogène...}

Oui, nous visons l'ensemble du corps médical mais, en fait, les conditions sont très différentes d'un professionnel à l'autre. Une partie de l'ancienne génération ne voudra sans doute plus participer au DEP. Si une per- 
sonne a prévu de prendre sa retraite dans cinq ans, je peux tout à fait comprendre qu'elle ne souhaite plus s'investir dans un tel projet. Par contre, il se pourrait bien qu'elle ait un intérêt à transmettre un cabinet qui soit techniquement à jour, ce qui engloberait, entre

\section{«Notre objectif premier est de donner envie à nos interlocuteurs de participer à la course.»}

autres, un dossier électronique pour chaque patient. C'est là-dessus que nous devrons nous concentrer ces prochains temps.

Et du côté des patients? Est-il vrai que seule une infime partie d'entre eux est informée sur le DEP?

Nous avons prévu de procéder par étapes. Les médecins seront les premiers visés, car ils constituent les premiers interlocuteurs des patients. Mais il va de soi que, dans un second temps, nous aborderons directement les patients pour les informer. Tous les renseignements de base concernant le DEP seront publiés sur le site www.dossierpatient.ch, l'idée étant de regrouper des contenus clairs, notamment sous la forme de vidéos. Une brochure explicative sera également éditée. Nous intensifierons la campagne d'information des patients ces prochains mois et une nouvelle fois au début de 2018.

Revenons-en à notre marathon. Quel est le rôle de la Confédération dans cette course? Est-elle le lièvre qui finit par s'effacer, l'organisateur chargé de définir le cadre et d'assurer un bon déroulement, ou porte-t-elle plusieurs casquettes?

La Confédération et les cantons sont les organisateurs. Et comme il s'agit d'une longue course, l'importance de l'organisation est immense. Notre objectif premier est de donner envie à nos interlocuteurs de participer à la course. Pour ce faire, la Confédération a notamment mis en place des incitations en proposant un soutien financier aux communautés et aux communautés de référence.

À ce propos, les communautés de référence recevront une aide financière, les cabinets médicaux, non. Pourquoi avoir pris une telle décision?

C'est vrai, c'est un sujet qui a fait l'objet de discussions au Parlement. Et c'est une décision politique qu'il nous faut accepter.

La Confédération joue en partie le rôle du lièvre. Les hôpitaux, par exemple, ont l'obligation de mettre en place le DEP dans un délai de trois ans.

Oui, c'est ce que le législateur a prévu pour les hôpitaux et les établissements médico-sociaux. Par ailleurs, les médecins tout comme les patients auront le choix de créer ou de gérer un dossier électronique.

\section{Comment allez-vous inciter les médecins à participer?}

De mon point de vue, l'information sera déterminante. Nous devons mettre en avant les avantages du DEP pour les patients mais également, à terme, pour les médecins. Il faudra notamment proposer des aides pour le lancement comme, par exemple, des solutions de connexion open source au DEP pour les systèmes d'information des cabinets médicaux et des cliniques.

Soyons un peu plus concrets. Comment le DEP peut-il encore améliorer le système suisse de santé, déjà très performant?

Pour les professionnels de la santé, l'avantage indéniable du DEP sera de pouvoir accéder en tout temps et en tout lieu aux informations sur leurs patients. Concrètement, cela facilitera la transmission des informations et permettra, par exemple, d'éviter des examens redondants.

\section{«Je pense que l'élément le plus important, c'est} la confiance dans le système.»

Les patients ne seront plus contraints d'emporter leurs radiographies, les rapports de sortie ou leurs ordonnances à chaque consultation. Toutes ces informations seront réunies dans le DEP. La sécurité des patients sera également renforcée puisque le médecin pourra immédiatement savoir quels médicaments prend le patient ou à quoi il est allergique. Notamment chez les patients polymorbides, suivis par plusieurs médecins qui ne savent pas toujours ce que leurs confrères ont prescrit.

\section{En théorie, le projet a l'air tout à fait convaincant. Comporte-t-il des facettes problématiques?}

Les médecins doivent être prêts à s'investir dans le projet. Et cela demande une certaine infrastructure. Il faut aussi que nous arrivions à bien informer les patients sur le DEP, surtout sur les aspects concernant la sécurité des données. Je pense que l'élément le plus important, c'est la confiance dans le système. Tous les participants, et surtout les patients naturellement, doivent pouvoir avoir la garantie que les données stockées dans le DEP ne seront pas utilisées de manière abusive. D’ailleurs ce sont eux qui décideront qui peut accéder à leurs données.

Selon vous, les problèmes ne sont donc pas liés au projet lui-même mais découlent plutôt de la motivation des acteurs?

Comme je viens de l'expliquer, il faut les deux: de la volonté et une infrastructure. 
Des membres de plusieurs groupes d'intérêt estiment que le projet a été développé selon une approche "topdown" très marquée.

Oui, j'ai entendu dire que ce sujet avait été abordé au fil de l'élaboration des ordonnances. Mais selon moi, les problèmes ont pu être résolus, et les acteurs concernés ont été consultés. J'irai même plus loin: le développement du DEP a été particulièrement marqué par la collaboration de tous les intéressés.

L'un des objectifs récurrents, lorsqu'on parle du DEP, c'est le renforcement de la culture sanitaire du patient. Dans quelle mesure le DEP contribue-t-il à atteindre cet objectif?

Il faut d'abord se mettre d'accord sur la définition de la culture sanitaire. Il s'agit, entre autres, de la capacité à se procurer des informations pertinentes, à les comprendre et à prendre ensuite des décisions concernant sa propre santé. Vu le volume d'informations et le nombre de canaux à disposition, les patients en savent

\section{"Je ne vois pas le DEP comme un problème, mais comme la solution.»}

toujours plus sur leur santé, leurs maladies. L'avantage du DEP, c'est qu'il réunit toutes les informations nécessaires au traitement. Comme le patient peut le consulter, il peut suivre les étapes principales de son parcours médical. Il peut également enregistrer ses propres données dans le dossier, ce qui sera de plus en plus utile au fil du temps. Au final, les médecins et les patients seront mieux préparés pour la consultation, et chacun pourra poser les bonnes questions de manière ciblée.

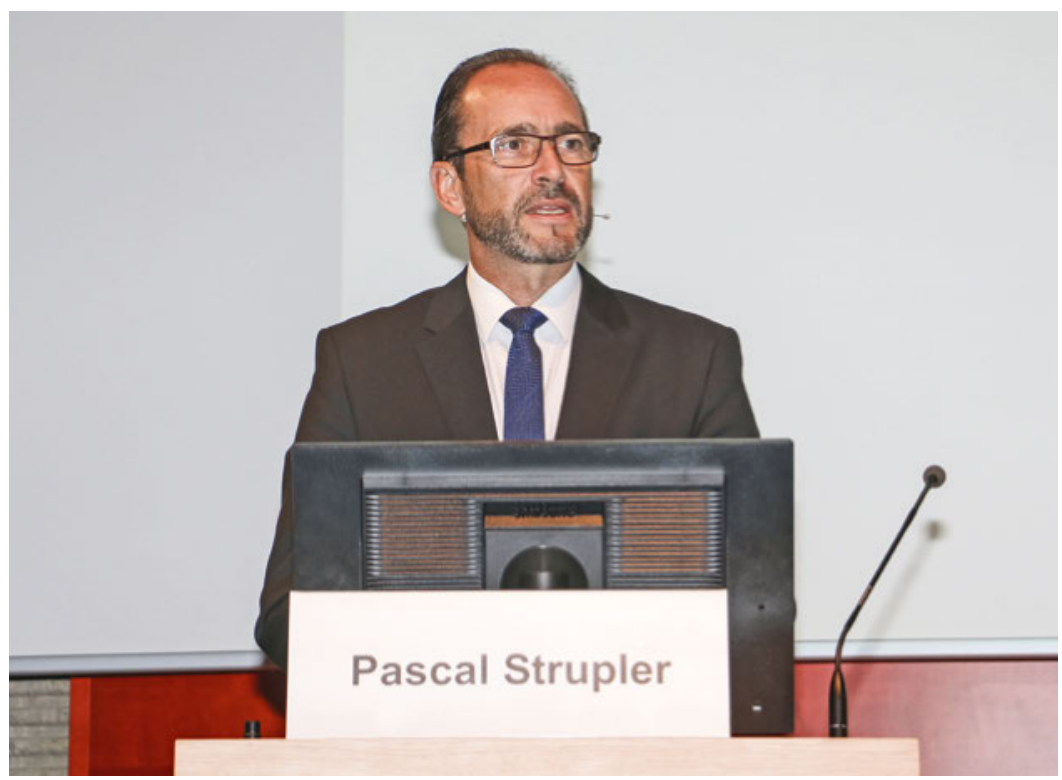

Pascal Strupler est convaincu des avantages du DEP.
Cela nous amène à nous interroger sur la qualité et la quantité des données qui seront enregistrées dans le DEP. Qui s'assure que les données stockées sont effectivement pertinentes et à jour?

Il n’y a pas de personne spécifiquement responsable. Un patient qui alimente lui-même le dossier est maître de ses données et responsable des contenus qu'il saisit. Du côté des médecins, il est légitime de penser que les enregistrements seront pertinents; et bien entendu, ils pourront toujours discuter avec les patients des informations placées dans leur dossier. Les informations devenues obsolètes ou inutiles pourront être supprimées d'un commun accord.

Vous ne trouvez pas cela problématique qu'un grand nombre d'acteurs soient impliqués dans le DEP? Je pense aux patients polymorbides, qui sont souvent suivis par plusieurs médecins ou thérapeutes et dans différents établissements.

Je ne vois pas le DEP comme un problème, mais comme la solution. Le cas de ces patients est bien réel, et le DEP leur permet justement, à eux et à leurs médecins, d'avoir une vue d'ensemble.

Le temps passé à gérer le DEP ne sera pas négligeable, notamment pour les cabinets des médecins de famille, qui en fin de compte devront probablement centraliser l'administration du dossier. Comment seront rémunérées ces prestations? Pourrait-on les comptabiliser comme "prestation médicale en l'absence du patient»?

Les travaux effectués sur le dossier médical du patient sont déjà rémunérés aujourd'hui.

S'il est bien une chose qui manque aux médecins, c'est le temps pour leurs patients. La situation ne risque pas de s'améliorer si chaque cabinet doit actualiser des milliers de DEP en plus des dossiers médicaux... Chaque changement, chaque nouvelle technologie à intégrer dans le travail quotidien demande un certain rodage et prend un peu plus de temps au début. Cet investissement, indéniable, est le lot de chaque branche professionnelle et peut légitimement être attendu de toutes les professions. L'effort à consentir et l'utilité du DEP doivent être soupesés non pas à court terme, mais dans une perspective plus longue. Bien organisé, le dossier électronique du patient permet, par exemple, aux médecins à qui on adresse un patient d'appréhender rapidement la situation. Ainsi, on évitera des examens redondants ou des demandes chronophages d'informations, pour ne nommer que ces avantages. À mon sens, tous les acteurs impliqués dans le traitement gagneront du temps à terme grâce au DEP. 
Prenons un patient un peu désorienté qui nécessite un traitement d'urgence. Le médecin peut-il être certain que le DEP contient bien les informations pertinentes et actualisées? Il se pourrait que le patient ait consulté un spécialiste peu avant et que ce praticien lui ait prescrit un nouveau médicament sans avoir eu le temps de l'enregistrer...

\section{«L'effort à consentir et l'utilité du DEP doivent} être soupesés dans une perspective plus longue.»

Je ne pense pas que le DEP reflète toute la réalité. Son efficacité dépendra des données saisies. Pas parfait, certes, mais il ne faut pas non plus poser des exigences irréalistes. Toutefois, le dossier électronique apportera des avantages substantiels dans la communication entre les différents acteurs qui s'occupent d'un patient. L'expérience faite par les pays nordiques le montre clairement.

\section{Ce projet soulève probablement aussi des questions juridiques. Est-ce que, par exemple, le médecin pourra effacer des saisies erronées? Qui sera habilité à "nettoyer» le DEP? Faudra-t-il une autorisation du patient? Qui sera tenu responsable pour une erreur médicale provoquée par une saisie erronée ou une lacune d'information, mettons sur une allergie? A-t-on pensé à ces aspects?}

Oui, de telles questions ont naturellement fait l'objet de vastes discussions pendant la procédure de consultation. De nombreux aspects juridiques comme la responsabilité civile ou le représentant du patient sont soumis aux mêmes règles que celles qui s'appliquent déjà. Il va sans dire que nous devrons gérer la mutation culturelle qu'entraînera le DEP. Mais, comme dit précédemment, personne ne chapeautera ce dossier électronique; par contre, le patient participera mieux au processus et sera davantage responsabilisé.

\section{"À l'heure actuelle, il n'est pas encore possible de projeter un bilan global.»}

\section{Comment estimez-vous l'impact du DEP sur les coûts de la santé? Entre-temps, il n'est presque plus question d'économies.}

Le dossier électronique du patient ne résoudra pas non plus tous les problèmes de coûts. Les examens redondants diminueront, c'est certain. Il en résultera non seulement des économies, mais aussi une plus grande efficacité, une meilleure sécurité pour le patient et, en fin de compte, un regain de qualité. Après, le DEP ne pourra pas empêcher un médecin de multiplier ses interventions. À l'heure actuelle, il n'est pas encore possible de projeter un bilan global.
Actuellement, on constate un grand clivage entre les différents groupes intéressés. Dans les branches techniques, on assiste à une espèce de "ruée vers l'or" tandis que chez les médecins, le scepticisme semble encore nettement prédominer.

J'ai une perception plus nuancée. Selon moi, le rapport du médecin au DEP est une question de génération. La motivation à se lancer varie énormément. Je pense que les associations de médecins doivent commencer à faire entendre leur voix auprès des prestataires de services informatiques pour leur communiquer leurs exigences et leurs préoccupations. L'OFSP, de son côté, s'attache d'ailleurs à les encourager sur cette voie.

\section{Alors, vous misez sur la nouvelle génération de praticiens pour le DEP?}

Pour les médecins actuellement assistants dans les hôpitaux, le travail avec des données électroniques est déjà monnaie courante. Chaque jour, ils en constatent les avantages et conserveront ce bagage lorsqu'ils s'installeront à leur tour. Par ailleurs, la tendance à ouvrir des cabinets de groupe permettant le travail à temps partiel appelle de telles infrastructures. En effet, il sera de plus en plus difficile de travailler «raisonnablement» sans recourir à la numérisation.

Il serait judicieux que les systèmes utilisés dans les cabinets puissent se connecter au DEP et que les données liées au diagnostic et aux médicaments soient mises à jour automatiquement. Quelles sont les perspectives ici?

C'est l'exemple par excellence d'une exigence que le corps médical peut faire valoir auprès des prestataires informatiques: une connexion sans accrocs de leurs systèmes informatiques au DEP. A cette fin, eHealth Suisse a déjà organisé des séances d'information et mis des outils à disposition. Face au vif intérêt de la branche informatique, je suis confiant.

\section{Si vous deviez faire un pronostic: quelle trajectoire traceriez-vous pour le projet du DEP?}

Dans la prochaine étape, les hôpitaux joueront un rôle important puisqu'ils ont l'obligation de mettre sur pied le DEP dans les trois ans à venir. Et, s'ils remplissent bien leur mission, le reste devrait s'enchaîner A mon avis, il est également crucial que, d'emblée, la sécurité des données ne pose aucun problème. Le DEP sera largement tributaire de la confiance des usagers, qui sont très sensibles à la sécurité des données sur la santé. 


\section{Le dossier électronique du patient (DEP)*}

Le dossier électronique du patient (DEP) est un ensemble de documents concernant une patiente ou un patient. Ces documents contiennent des informations pertinentes pour le traitement telles que le rapport de sortie de I'hôpital, le rapport de soins à domicile, les radiographies, le carnet de vaccination ou l'ordonnance pour la pharmacie. Les patientes et patients peuvent aussi ajouter au DEP leurs propres informations de santé comme l'ordonnance pour les lunettes, leurs directives anticipées ou leur pression artérielle.

Pour lire les documents se trouvant dans le DEP, les professionnels de la santé doivent avoir un droit d'accès explicite. Celui-ci leur est octroyé par la patiente / le patient. La possibilité de consulter des documents sans droit d'accès n'est possible qu'en cas d'urgence. La patiente / le patient doit ensuite être informé-e de cet accès.

Le dossier électronique du patient (DEP) est un regroupement de différents projets autonomes. Dans ce cadre, des professionnels de la santé et leurs établissements s'organisent en groupements organisés sur le plan technique. De tels groupements sont nommés «communautés du DEP».

Dans certaines communautés, le patient peut ouvrir son DEP personnel. De telles communautés sont nommées "communautés de référence». Les communautés et communautés de référence peuvent se former de manière décentralisée dans des régions de fourniture des soins, par exemple dans un canton ou de manière à englober plusieurs cantons. Tous les professionnels de la santé et leurs établissements peuvent s'y affilier (par exemple hôpitaux, établissements médico-sociaux, cabinets médicaux, pharmacies ou services de soins à domicile).

Informations détaillées au sujet du DEP: www.dossierpatient.ch 